\title{
Effects of Gravity-Assist Timing on Outer-Planet Missions Using Solar-Electric Propulsion
}

\author{
Byoungsam Woo* and Victoria L. Coverstone ${ }^{\dagger}$ \\ University of Illinois at Urbana-Champaign, Urbana, Illinois, 61801 \\ and \\ Michael Cupples ${ }^{\ddagger}$ \\ Science Applications International Corporation, Huntsville, Alabama, 35806
}

\begin{abstract}
Missions to the outer planets for spacecraft with a solar-electric propulsion system (SEPS) and that utilize a single Venus gravity assist are investigated. The trajectories maximize the delivered mass to the target planet for a range of flight times. A comparison of the trajectory characteristics (delivered mass, launch energy and onboard propulsive energy) is made for various Venus gravity assist opportunities. Methods to estimate the delivered mass to the outer planets are developed.
\end{abstract}

\section{Nomenclature}

$C=$ linear least square constant

c = magnitude of exhaust velocity

$C_{3}=$ square of the earth-centered hyperbolic excess velocity

$\Delta E \quad=$ total propulsive specific energy increment

$\Delta V_{\text {SEPS }}=$ velocity increment provided by onboard SEPS

$g=$ gravitational acceleration at the surface of the Earth

$I_{s p_{*}} \quad=$ specific impulse

$I_{s p} \quad=$ average specific impulse of a SEPS trajectory

$I_{s p}{ }^{m} \quad=$ median of $I_{s p}{ }^{*}$ range in all trajectories of this research

$k=$ linear least square scale factor

$m_{0}, m_{F}=$ initial injected mass and delivered mass

$\dot{m} \quad=$ mass flow rate

$\xi$, TOF $=$ time of flight

\section{Introduction}

TN exploring the outer solar system planets, a planetary gravity-assist has commonly been used since one or more I gravity-assists have the potential to save propellant, reduce time of flight (TOF), or both. Because of these advantages, many previous interplanetary missions (for example, Mariner 10, Voyager I, II, Galileo, Cassini and NEAR) exploited the gravity-assist. ${ }^{1}$ However, for a ballistic interplanetary mission, there are very little chances that all flyby planets are in the desired positions at the desired time. Commonly, the launch may have to be postponed by a couple of years if a few-day launch opportunity is missed. Moreover, this short launch opportunity shrinks rapidly as the number of flyby planets increases. Solar Electric Propulsion Systems (SEPS) can provide longer launch opportunities than a ballistic mission. The trajectory shaping capability of SEPS as well as the longer launch opportunity increases mission design flexibility.

* Graduate Research Assistant, Department of Aerospace Engineering, 326 Talbot Lab. 104 S. Wright St., Student Member AIAA.

${ }^{\dagger}$ Associate Professor, Department of Aerospace Engineering, 306 Talbot Lab. 104 S. Wright St, Associate Fellow AIAA.

${ }^{\ddagger}$ Lead Systems Engineer, In-Space Technology Assessment, National Space Science \& Technology Center, 320 Sparkman Dr.. 
The flexibility of SEPS missions also allows the gravity-assist to occur at different times even with the same TOF and the same launch year constraints. In a previous study, ${ }^{2}$ we found that the different gravity-assist timing changes the mission performance in terms of delivered mass to target planets in outer-planet, single Venus gravityassist missions. Revolution ratio (R-ratio) was introduced to represent the different Venus gravity-assist timing and was defined as the number of Venus revolutions for one revolution of a spacecraft around the Sun. For instance, a 3:1 R-ratio is one where roughly three Venus years occur during the period from launch to flyby of Venus by the spacecraft. For a SEPS mission, the R-ratio can be considered as the problem of trade-off between the launch energy and the onboard propulsive energy or similarly, the problem of allocating the flight time to before and after of the gravity-assist. For this study, we used SEPTOP (Solar Electric Propulsion Trajectory Optimization Program) ${ }^{3}$ to generate SEPS, outer-planet optimal trajectories. In spite of the necessity of the R-ratio analysis, it can be difficult to obtain the different R-ratio optimal trajectories with SEPTOP. In this paper, the R-ratio problem is revisited to clarify the underlying fundamentals of the problem and methods to estimate the delivered mass of the different $R$ ratio trajectories are developed to support the $\mathrm{R}$-ratio analysis.

\section{Revolution Ratio}

Figure 1 illustrates the trajectories in an Earth-Venus-Saturn (EVS) mission with three different R-ratios. The TOF of all trajectories in the figure is 6 years. It is clear that a spacecraft on the trajectory with the largest R-ratio spends the most time thrusting before the flyby occurs. Given the nature of SEPS performance - namely, that orbital energy addition is more efficient near the Sun due to greater power availability and control authority - it would seem that spending more time in close proximity to the Sun before heading outbound toward the destined target would be beneficial in delivering more mass. However, a larger R-ratio trajectory typically needs more launch energy, which in turn means a larger proportion of the total required energy being provided by an inefficient launch vehicle rather than the more efficient low-thrust engine. Trade-offs between the launch energy and the onboard propulsion result in an optimal R-ratio of 2:1 for this TOF 6 year mission. Here, the launch energy is provided by Delta IV $\mathrm{M}+(4,2)$ launch vehicle and High Thrust To Power 3900 s (HTTP 3900 ) thruster ${ }^{4}$ is used as onboard propulsion system.

Figure 2 shows the delivered mass comparison for the EVS, Earth-Venus-Uranus (EVU), EarthVenus-Neptune (EVN), and Earth-Venus-Pluto (EVP) missions. This comparison is a result of the launch energy and onboard propulsion trade-off. A more detailed comparison can be found in Ref. 2 .

\section{SEPS Thruster Model}

The free and continuous thrust profile of SEPS provides serious difficulties in analytical trajectory design. In this paper, a thruster model that represents the overall thrust profile is developed to be exploited in the delivered mass estimation of SEPS trajectories. The thruster model includes constant thrust, mass flow rate and $I_{s p}$ or exhaust velocity $c$. These parameters are varying during a mission and the variation is mission specific. Because of the variable characteristics of a SEPS, the established rocket equation is not applicable for a SEPS mission. With constant parameters of a SEPS thruster, it would be possible to use the rocket equation to estimate the delivered mass of the mission. The SEPS thruster model is a function of the input power so the modeling of the constant thruster parameters is

HITP3900, EVS. 4 Engine Case

6 year TOF

Power into thrusters $=23.3825 \mathrm{~kW}$

Total Mass Delivered $(2-1)=2493.01 \mathrm{~kg}$

Total Mass Delivered (3:1) $=2467.69 \mathrm{~kg}$

Total Mass Delivered $(4: 1)=2226.53 \mathrm{~kg}$

Propellant Mass $(2 \div 1)=978.69 \mathrm{~kg}$

Propellant Mass (3:1) $=741.79 \mathrm{~kg}$

Propellant Mass $(4: 1)=640.68 \mathrm{~kg}$ Delta IV M+(4,2)

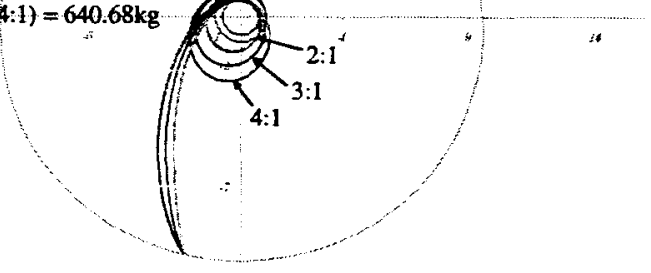

Figure 1. Trajectories with three different R-ratios for an EVS mission, Delta-IV M+ (4,2).

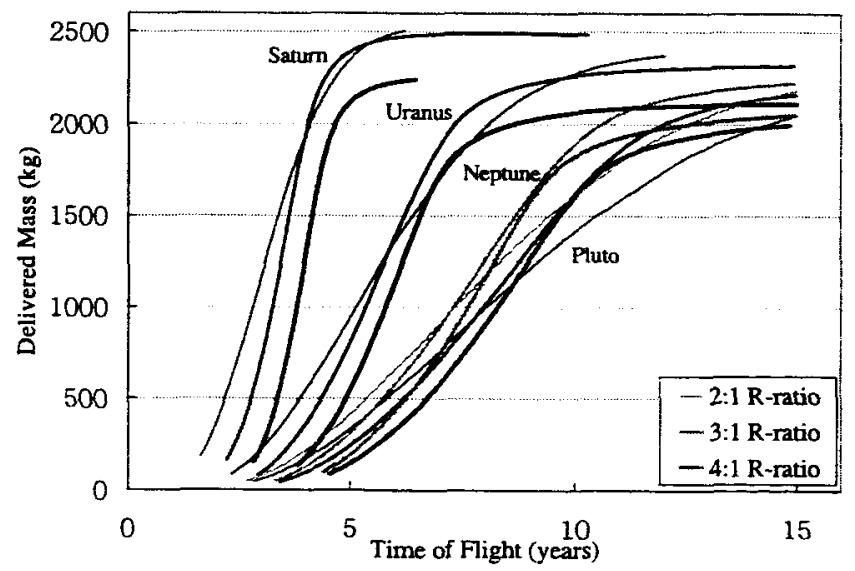

Figure 2. Delivered mass of three different R-ratios: EVS, EVU, EVN, and EVP missions. 
equivalent to the selection process of the effective input power $\left(\mathrm{P}_{\text {eff }}\right)$ within the operating power range. Once the $P_{\text {eff }}$ is determined, the thrust and mass flow rate are calculated from the original thruster model. ${ }^{4}$ In case the duty cycle of the SEPS is not $100 \%$, then the calculated thrust and mass flow rate are reduced by multiplying by the duty cycle percentage. For multiple thrusters, the number of thrusters is multiplied to the thrust and mass flow rate to find the total thrust and mass flow rate for a mission. With these results, the mission-specific overall $I_{s p}$ (or magnitude of exhaust velocity $c$ ) is calculated by Eq. (1). The $\dot{m}$ is mass flow rate and $g$ is gravitational acceleration at the surface of the Earth.

$$
I_{s p}=\text { thrust } / \dot{m g}=c / g
$$

If we define $\mathrm{I}_{\mathrm{sp}}{ }^{*}$ as an average mission-specific $\mathrm{I}_{\mathrm{sp}}$, the $\mathrm{I}_{\mathrm{sp}}{ }^{*}$ is calculated by the initial mass that is inserted to orbit by a launch vehicle $m_{0}$, the delivered mass $m_{F}$ and the velocity increment provided by SEPS during the flight $\Delta V_{S E P S}$ from SEPTOP by Eq. (2) (Ref. 5).

$$
I_{s p}^{*}=\frac{-\Delta V_{S E P S}}{g \cdot \ln \left(m_{F} / m_{0}\right)}
$$

The $\mathrm{I}_{\mathrm{sp}}{ }^{*}$ represents the averaged effect of the thrusting in a trajectory. For all outer-planet trajectories of this research, the $I_{s p}{ }^{*}$ varies between $3166.357 \mathrm{~s}$ to $3628.537 \mathrm{~s}$. The median of the $I_{s p}{ }^{*}$ variation $I_{s p}{ }^{m}$ is $3397.4 \mathrm{~s}$ and it is $90 \%$ of the maximum $I_{s p}$ of the HTTP 3900 thruster. The $P_{\text {eff }}$ that generate $I_{s p}{ }^{m}$ of $3397.4 \mathrm{~s}$ is calculated from the HTTP 3900 thruster characteristics and it results $P_{\text {eff }}$ of $4.32 \mathrm{kWe}$. It is approximately $75 \%$ of the maximum operating power of the thruster. The $\mathrm{I}_{\mathrm{sp}}{ }^{\mathrm{m}}$ (or $c$ ) is the representative value for a range of actual $\mathrm{I}_{\mathrm{sp}}$ 's so there exists differences between the $I_{s p}{ }^{m}$ and the actual $I_{s p}$ 's. The difference can be as large as 230 sec in this research so the validity of this modeling strategy should be reviewed before using the $I_{s p} m$. The validity of the $I_{s p}{ }^{m}$ as a representative value for a SEPS thruster can be shown by examining the sensitivity of the delivered mass $\left(m_{F}\right)$ to the variation of $\mathrm{I}_{\mathrm{sp}}$. In order to see this, consider the solution to the rocket equation in Eq. (3) (Ref. 5).

$$
\exp \left[-\Delta V_{S E P S} / c\right]=\exp \left[-\Delta V_{S E P S} / g I_{s p}\right]=m_{F} / m_{0}
$$

Examining first-order variations in $\mathrm{I}_{\mathrm{sp}}$ and $m_{F}$.

$$
\exp \left[\frac{-\Delta V_{\text {SEPS }}}{g I_{s p}}\right] \cdot\left(\frac{-\Delta V_{\text {SEPS }}}{g} \frac{-d I_{s p}}{I_{s p}{ }^{2}}\right)=\frac{\Delta V_{\text {SEPS }}}{g I_{s p}{ }^{2}} \cdot \exp \left[\frac{-\Delta V_{S E P S}}{g I_{s p}}\right] \cdot d I_{s p}=\frac{d m_{F}}{m_{0}}
$$

which can be expressed using Eq. (2) as

$$
\frac{\Delta V_{S E P S}}{g I_{s p}} \frac{d I_{s p}}{I_{s p}}=\frac{d m_{F}}{m_{F}}
$$

In Eq. (5), $\Delta V_{S E P S} / g I_{s p}$ is typically a number less than one. For a typical EVS mission, $\Delta V_{S E P S} / g I_{s p}$ is about $3 \times 10^{-1}$. For sizable delivered masses of $\mathrm{m}_{\mathrm{F}} \approx 2000 \mathrm{~kg}$, this makes $d m_{F} / m_{F}$ relatively insensitive to $d I_{s p} / l_{s p}$ in a SEPS mission. On the other hand, for a chemical engine mission with the similar $\Delta V$, the $d m_{F} / m_{F}$ is more sensitive to $d I_{s p}$ than for a SEPS mission because of the smaller $I_{\mathrm{sp}}$ range of 200 to $410 \mathrm{~s}$ (Ref. 6).

If the change of $\mathrm{I}_{\mathrm{sp}}$ is so large that the first-order analysis is not accurate, one must compare the nonlinear values directly. Starting from Eq. (2), let

$$
\frac{d m_{F 1}}{m_{0}}=\exp \left[\frac{-\Delta V_{S E P S}}{g I_{\text {sp } 1}}\right]
$$




$$
\frac{d m_{F 2}}{m_{0}}=\exp \left[\frac{-\Delta V_{S E P S}}{g I_{s p 2}}\right]
$$

and define

$$
\begin{aligned}
& \Delta m_{F}=m_{F 2}-m_{F 1} \\
& \Delta I_{s p}=I_{s p 2}-I_{s p 1}
\end{aligned}
$$

then

$$
\frac{\Delta m_{F}}{m_{0}}=\frac{m_{F 2}}{m_{0}}-\frac{m_{F 1}}{m_{0}}=\exp \left[\frac{-\Delta V_{S E P S}}{g I_{s p 2}}\right]-\exp \left[\frac{-\Delta V_{S E P S}}{g I_{S P 1}}\right]
$$

and finally,

$$
\begin{aligned}
\frac{\Delta m_{F}}{m_{F 1}} & =\frac{\exp \left[\frac{-\Delta V_{S E P S}}{g I_{s p 2}}\right]-\exp \left[\frac{-\Delta V_{S E P S}}{g I_{s p 1}}\right]}{\exp \left[\frac{-\Delta V_{S E P S}}{g I_{p 1}}\right]}=\exp \left[\frac{-\Delta V_{S E P S}}{g I_{s p 2}}+\frac{\Delta V_{S E P S}}{g I_{s 1}}\right]-1 \\
& =\exp \left[\frac{-\Delta V_{S E P S}}{g}\left(\frac{I_{s p 1}-I_{\$ p 2}}{I_{s p 1} I_{\$ p 2}}\right)\right]-1=\exp \left[\frac{\Delta V_{S E P S}}{g} \cdot \frac{\Delta I_{s p}}{I_{s p 1} I_{s p 2}}\right]-1
\end{aligned}
$$

The representative $I_{s p}{ }^{m}$ value for the HTTP 3900 thruster is $3397.447 \mathrm{~s}$ and the variation of $I_{s p}{ }^{*}$ in all trajectories with various TOF is $\mathrm{I}_{\mathrm{sp}}{ }^{\mathrm{m}} \pm 230 \mathrm{~s}$. The maximum $\Delta m_{F} / m_{F I}$ deviation for the range of $\mathrm{I}_{\mathrm{sp}}{ }^{\mathrm{m}}$ is less than $5 \%$ for $\Delta V_{\text {SEPS }}=20$ $\mathrm{km} / \mathrm{s}$ case. In Fig. 3 the variation of $m_{F}$ is shown for the variation of $I_{s p}{ }^{*}$ near the representative $I_{s p}{ }^{m}$ value. In the figure, the maximum $m_{r}$ estimation error caused by the $I_{s p}{ }^{m}$ modeling inaccuracy is $-4.8 \%$ of the $m_{F}$ which is acceptable error for preliminary mission planning. In summary, the delivered mass is not highly sensitive to the specific impulse modeling inaccuracy for the SEPS outerplanet missions and therefore, a constant specific impulse can be used for several mission planning purposes.

\section{Estimation of Delivered Mass of Different R-ratio Trajectories}

\section{A. Estimation of Delivered Mass with Linear Least Square Method}

The bases of the delivered mass estimation method are the regularities of the relation between the delivered mass and the total specific propulsive energy increment.

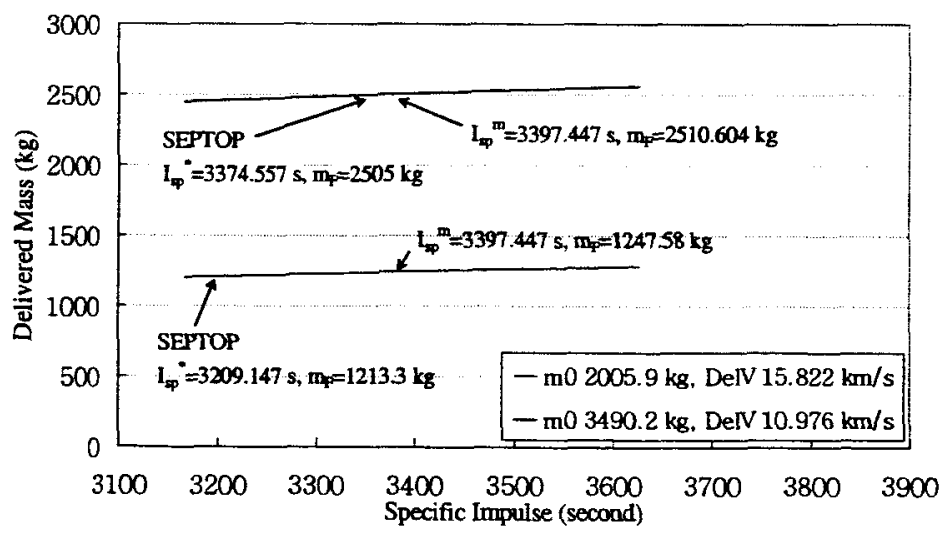

Figure 3. Delivered mass sensitivity to the specific impulse variation.

The total propulsive specific energy increment is defined as

$$
\Delta E=\Delta V_{S E P S}^{2}+C_{3}
$$


where $\Delta E$ is the total propulsive specific energy increment (excluding an energy increment by a gravity assist) and the launch energy $C_{3}$ is the square of the earth-centered hyperbolic excess velocity of a spacecraft when it has just separated from the launch vehicle. Figure 4 shows the $\Delta E$ of three R-ratio trajectories for EVS to EVP missions. In

Fig. 4 , the $\Delta E$ curves for a target planet have similar shape regardless of the different R-ratio. In other words, $\Delta E$ curves with different $\mathrm{R}$ ratios are related by a shift along with the TOF axis and the $\Delta E$ axis. The amount of the horizontal shifts (along with the TOF axis) is investigated to determine one Venus year (224.701 days) and the amount of the vertical shifts (along with the $\Delta E$ axis) is found to be 30 $\mathrm{km}^{2} / \mathrm{s}^{2}$ between $3: 1$ and $2: 1$ R-ratios and 15 $\mathrm{km}^{2} / \mathrm{s}^{2}$ between 4:1 and 3:1 R-ratios in Fig. 4. Figure 5 compares the $2: 1$ and $4: 1 \mathrm{R}$-ratio $\Delta E$ curves from SEPTOP with the shifted 3:1 Rratio $\Delta E$ curve for EVS mission. Overall, the shifted curve matches 2:1 and 4:1 R-ratio $\Delta E$ curves accurately. This result means that the $\Delta E$ curves with different $\mathrm{R}$-ratio to a target planet have similar profiles in spite of their different delivered mass profile. There are many potential applications of this result; for example, it is possible to predict the delivered mass of a mission with different R-ratio. Also, the search space for optimization can be dramatically reduced by previewing the delivered mass profiles of the different R-ratio trajectories and selecting the most promising R-ratio before starting the optimization. The comparison results for EVU, EVN and EVP missions are not included in this paper for conciseness but their results are similar with Fig. 5.

Another result that should be mentioned is that the horizontal distance between the curves is constantly one Venus year in all missions. Together with the definition of R-ratio, it is an interesting result that explains the physical differences between the different R-ratio trajectories. The difference in TOF from the launch date to the flyby date between the different R-ratio trajectories is roughly one Venus year by the definition of R-ratio so, in the point of $\Delta E$, the effect of the different Rratio is that a very similar $\Delta E$ curve is repeated with the TOF difference of one Venus year. This result provides a method to generate a delivered mass profile of a different R-ratio trajectory without actually calculating it by SEPTOP reducing time to provide preliminary mission planning results. In generating a delivered mass profile from a $\Delta E$ curve, the relation between the $\Delta E$ curve and the delivered mass profile needs to be established.

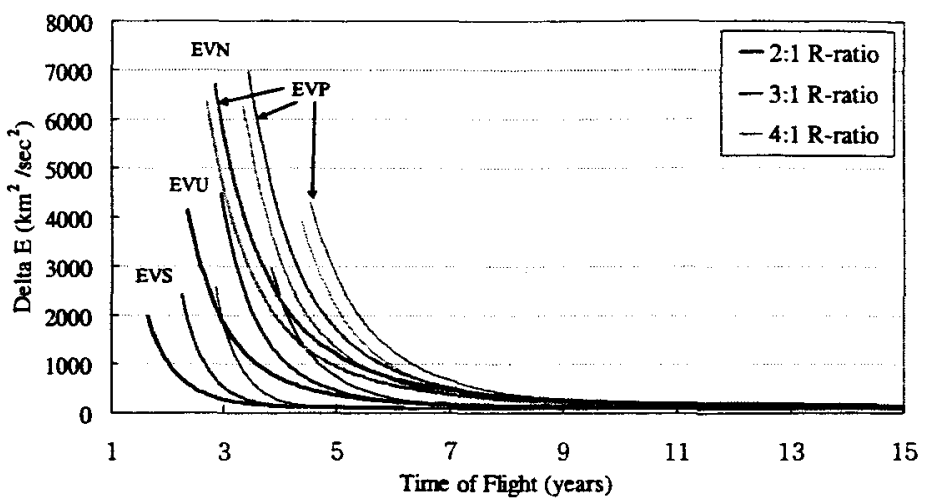

Figure 4. Total propulsive energy increment versus Time of flight.
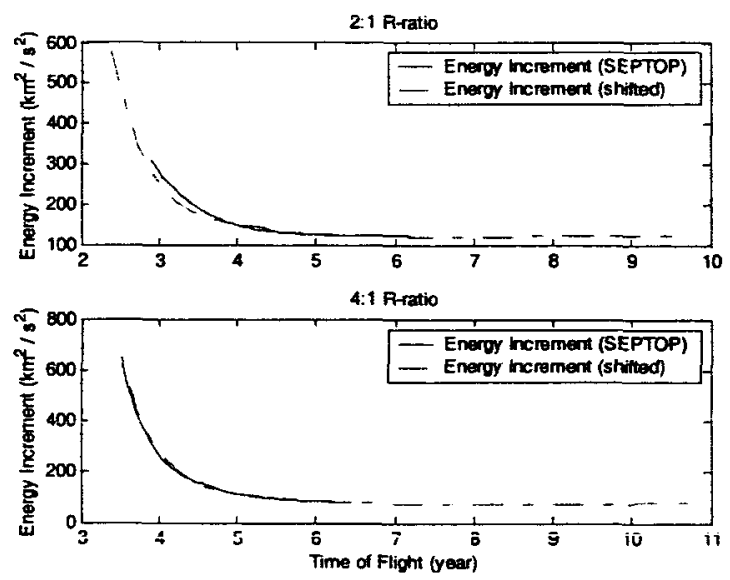

Figure 5. Total propulsive energy increment versus Time of flight.
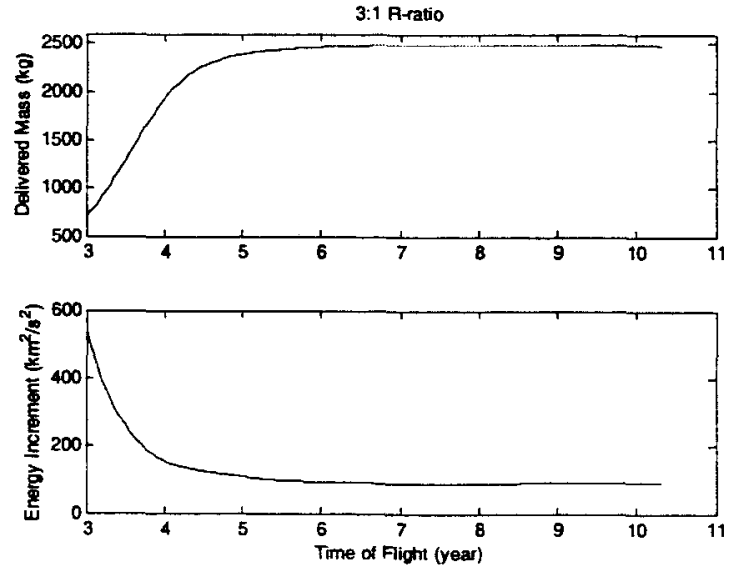

Figure 6. Delivered mass and $\Delta E$ for 3:1 R-ratio, EVS mission. 
Figure 6 shows the delivered mass and $\Delta E$ for 3:1 R-ratio, EVS mission. The delivered mass generation method is based on the symmetry between the $\Delta E$ and the delivered mass relative to a horizontal line in Fig. 6 . Because of

Table 1. $k$ and $C$ for EVS to EVP missions

\begin{tabular}{llll}
\hline Target & R-ratio & $k\left(\mathrm{~kg}^{\prime} \mathrm{km}^{2} / \mathrm{s}^{2}\right)$ & $C(\mathrm{~kg})$ \\
\hline Saturn & $2: 1$ & 8.501 & 3374.258 \\
& $3: 1$ & 5.154 & 2928.289 \\
Uranus & $4: 1$ & 3.579 & 2481.234 \\
& $2: 1$ & 4.908 & 2800.503 \\
Neptune & $3: 1$ & 3.866 & 2616.894 \\
& $4: 1$ & 2.898 & 2329.991 \\
& $2: 1$ & 4.163 & 2592.702 \\
Pluto & $3: 1$ & 3.594 & 2506.627 \\
& $4: 1$ & 2.592 & 2269.293 \\
& $2: 1$ & 3.964 & 2584.694 \\
& $3: 1$ & 3.414 & 2492.664 \\
\hline
\end{tabular}

their symmetry, the delivered mass profile can be represented by $\Delta E$ with a scale factor and a constant. Let $f_{l}(\xi)$ be the delivered mass function and $f_{2}(\xi)$ be the $\Delta E(\xi)$ function where $\xi$ is the time of flight. Then,

$$
f_{1}(\xi)+k \cdot f_{2}(\xi)=C
$$

where $k$ is a scale factor between the delivered mass and $\Delta E$, and $C$ is the representing constant for the trajectories to a target planet with the R-ratio. Equation (12) is rearranged to be used in linear least square fitting in the below equation.

$$
k \cdot f_{2}(\xi)-C=-f_{l}(\xi)
$$

For the given TOF $\xi_{i}, i=1, \ldots, n, \mathrm{Eq}$. (13) can be used to construct below linear least square equation.

$$
\left[\begin{array}{cc}
f_{2}\left(\xi_{1}\right) & -1 \\
\vdots & \vdots \\
f_{2}\left(\xi_{n}\right) & -1
\end{array}\right]\left(\begin{array}{l}
k \\
c
\end{array}\right)=-\left(\begin{array}{c}
f_{1}\left(\xi_{1}\right) \\
\vdots \\
f_{1}\left(\xi_{n}\right)
\end{array}\right)
$$

The Q-R factorization ' is used in solving Eq. (14) to calculate the $k$ and $C$. From $\Delta E$ 's of three R-ratios for EVS to EVP missions, the $k$ 's and $C$ 's are calculated and shown in Table 1.

The thruster used is HTTP 3900 and the launch vehicle is Delta IV $\mathrm{M}+(4,2)$. The $k$ and $C$ depend on the thruster and the launch vehicle because the relation between the $\Delta E$ and the delivered mass is determined by them. The $k$ represents the propulsion efficiency of $\Delta E$ to the delivered mass. Large $k$ means more efficient thrusting (launch vehicle + SEPS) for a given delivered mass. The unit of $k$ is $\mathrm{kg} \cdot \mathrm{s}^{2} /$ $\mathrm{km}^{2}$. The constant $C$ can be interpreted as a limit of the maximum delivered mass of the $\mathrm{R}$ ratio trajectory class to a target planet. According to Table 1, $k$ 's are larger for closer targets and that can be interpreted to mean that the thrusting is more efficient than for a farther targets. At the same time, $C$ 's for farther targets are smaller than $C$ 's for closer targets so the

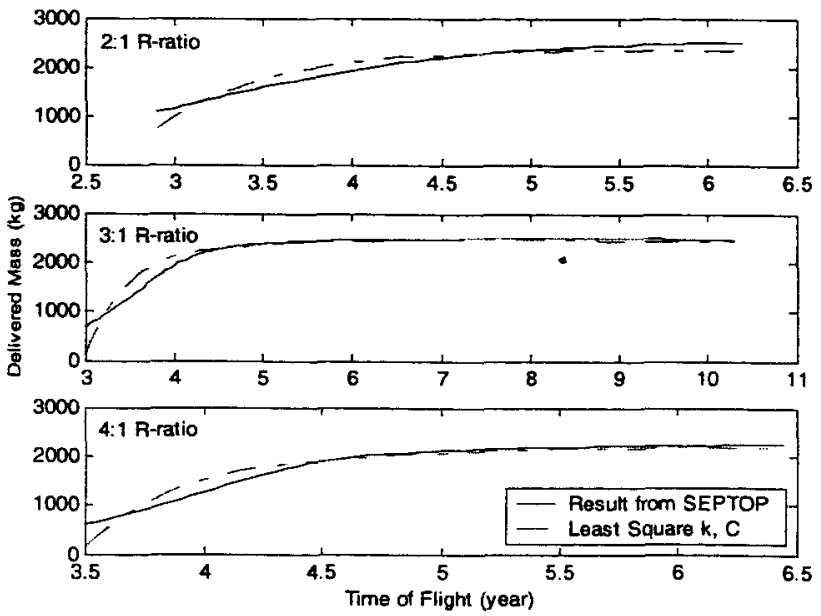

Figure 7. Delivered mass comparison between SEPTOP and $k$, $C$ fitting for EVS mission. 
limit of the delivered masses for farther targets are smaller. In Eq. (12), the $k$ and $C$ are regulating each other in the sense of increasing or decreasing the delivered mass to a target planet. The delivered mass is determined by the interplay between the $k$ and $C$ and the given profile of $\Delta E$ as a function of TOF.

In order to check the validity of $k$ and $C$, the delivered mass profiles are regenerated using Eq. (13) and $k$ and $C$ of Table 1. Figure 7 shows the comparison of the delivered masses from SEPTOP with the delivered mass from $k, C$ and $\triangle E$ from SEPTOP for an EVS mission. The overall predictive performance is observed in the figure. These results mean that if the $k, C$ and $\Delta E$ were known for a mission, the delivered mass profile for the mission could be estimated without using SEPTOP. As previously explained, the $\Delta E$ curves for a target planet can be approximated by a planar shift of a known $\Delta E$ curve. At present, a $\Delta E$ curve to a target planet is required to be calculated by SEPTOP to approximate the $\Delta E$ of the different R-ratio trajectories to the target planet. On the other hand, the $k$ and $C$ for a mission can be closely approximated once the $k$ and $C$ to a different target planet but with the same R-ratio are known from the SEPTOP result. In other words, $k$ and $C$ of a mission to a target planet with an $\mathrm{R}$-ratio have a relation with the $k$ and $C$ of a mission to a different target planet with the same R-ratio. The relation between $k$ 's and $C$ 's with the different $\mathrm{R}$-ratio to a target planet needs more research to be clarified. The relations between $k$ 's and $C$ 's of different missions are shown in Figs. 8 and 9 as functions of inverse of the semimajor axis of target planets. In the figures, the $k$ 's and $C$ 's are shown as linear functions of the inverse of semimajor axis. It is also possible to calculate $k$ 's and C's for other target planets if one use the linear functions and then possible to estimate the delivered mass to the target planets. In summary, Fig. 10 illustrates a procedure of linear least square method.

Figure 11 and Fig. 12 shows the comparisons between the estimated delivered mass and the delivered mass from SEPTOP for

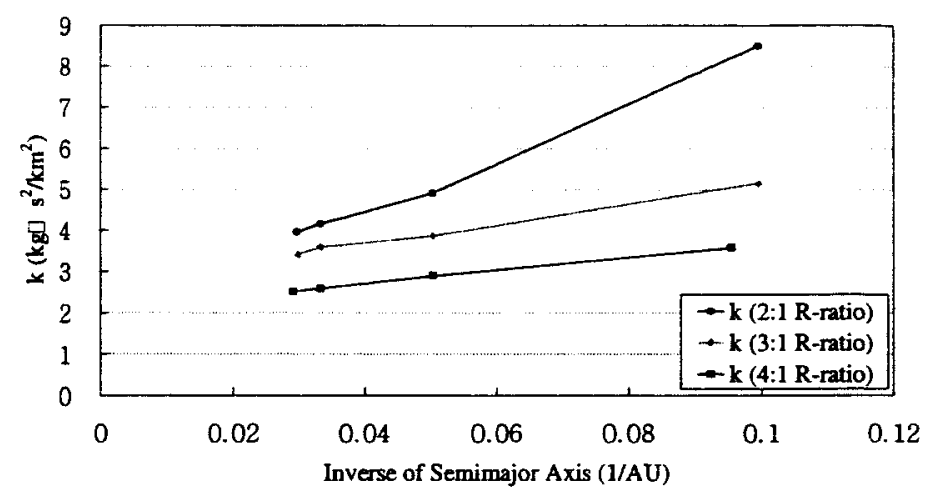

Figure 8. $\boldsymbol{k}$ variation as a function of inverse of semimajor axes.

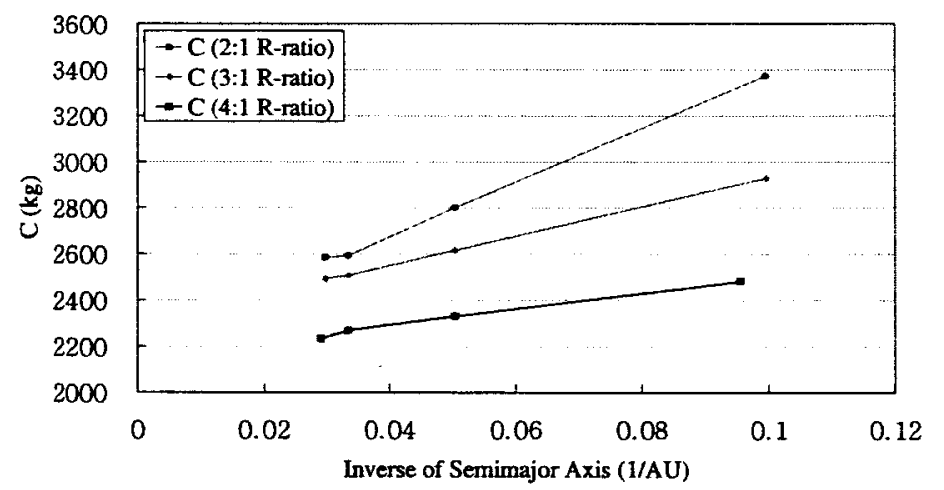

Figure 9. C variation as a function of inverse of semimajor axes.

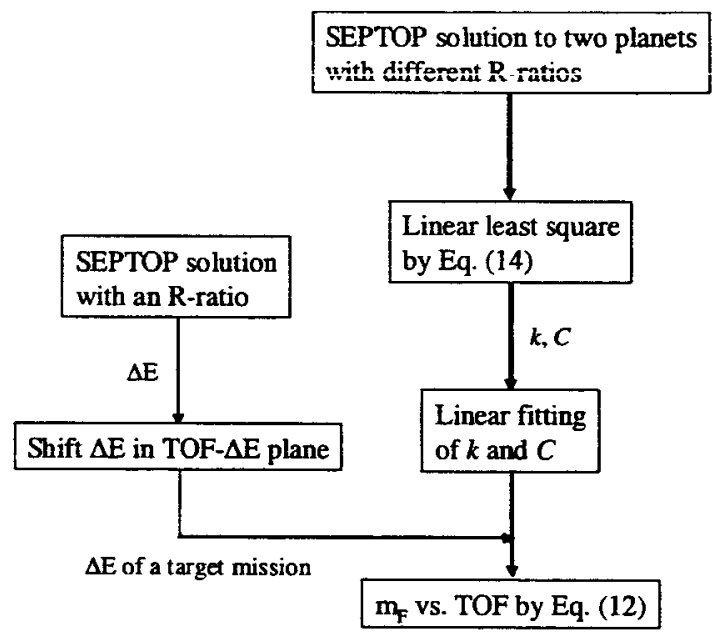

Figure 10. A procedure of linear least square estimation method.

EVS and EVP missions. The estimated delivered mass is calculated with $k$ and $C$ and planar shifted $\triangle E$ curves. EVU and EVN missions show similar results so they are excluded for the conciseness of the paper.

In Figs. 11 and 12, there are some differences between the regenerated delivered mass and the SEPTOP result in spite of the overall coincidences. The differences in short TOF range are caused by the incomplete symmetry between the delivered mass profile and the $\Delta E$ profile. The incompleteness is more outstanding in all of the 2:1 $\mathrm{R}$ ratio cases than the other cases and this is the reason of the relatively larger differences in short TOF, 2:1 R-ratio cases. The source of the differences in intermediate to long TOF range is the linear least square error. The linear 
least square algorithm tries to minimize the total error in all of the data range so a small range of large error usually degrade the total accuracy and the error spread out over all the data range. Therefore, there are relatively larger errors in long TOF, 2:1 R-ratio cases than in other R-ratio cases. The maximum difference between the regenerated delivered mass and the SEPTOP result is about $500 \mathrm{~kg}$ in short TOF. This estimated delivered mass can be used for some mission planning purposes, because the estimated delivered mass curve is a good approximation for middle to long TOF range missions, however, a more precise method to predict the delivered mass is required to determine which R-ratio trajectory is the best candidate for a given TOF range of interest without spending lots of effort in SEPTOP.

\section{B. Estimation of Delivered Mass with Hybrid Method}

The linear least square estimation method has some amount of error especially in its short TOF ranges. The error is caused by the incomplete symmetry between the delivered mass profile and the $\Delta E$ profile. In spite of the incomplete symmetry, however, the $\Delta E$ profile has significant advantage because the similarity in $\Delta E$ profiles among the different $\mathrm{R}$-ratio cases provides a basis to predict the delivered masses of the different R-ratio cases. A more precise estimation method, a hybrid method, also relies on the similarity of $\Delta E$ profiles as the linear least square method but it also employs a method that uses the thruster model and rocket equation.

In the hybrid method, the TOF range is divided to two ranges, short TOF and remaining TOF range, and a method that uses the rocket equation is applied to that short TOF range whereas the linear least square method that uses $k$ and $C$ is applied to the remaining TOF range. First, a solution to the rocket equation is shown below. ${ }^{5}$

$$
m_{F}=m_{0} \exp \left(\frac{-\Delta V_{\text {SEPS }}}{c}\right)
$$

The delivered mass can be calculated given $m_{0}, \Delta V_{S E P S}$ and $c$. The way to determine the $m_{0}$ and $\Delta V_{S E P S}$ for a given TOF is explained below and the $c$ is modeled by a constant $I_{s p}$ SEPS model.

In estimating the delivered mass, the given data are the $\Delta E$ and the delivered mass profile of an $\mathrm{R}$-ratio case (3:1 R-ratio case in this research). By a planar shift, the $\Delta E$ profile of $2: 1$ and $4: 1$ R-ratio cases can be approximated. However, it is required to find the $\Delta V_{S E P S}$ out of $\Delta E$ because Eq. (15) needs $\Delta V_{S E P S}$ to calculate the $m_{F}$ and $\triangle E$ is the sum of the $C_{3}$ and the square of $\Delta V_{S E P S}$. Of course, the $\Delta V_{S E P S}$ and also $C_{3}$ are assumed to be known for an R-ratio case in order to calculate the $\Delta E$ profile that is to be shifted. Therefore, the $C_{y} / \Delta E$ profiles of all R-ratio cases are compared to find the relation between them. Figure 13 shows the variation of $C_{3} / \Delta E$ versus $\Delta E$ profile for the short TOF range, for all target missions. By comparing the $\Delta E$ values with Fig. 4 , one can notice that the TOF gets shorter 
as $\Delta E$ value increases in Fig. 13. The $C_{3} / \Delta E$ profiles are generated with SEPTOP results and almost identical in all R-ratio missions. This fact provides a way to find the $\Delta V_{\text {SEPS }}$ out of the shifted $\Delta E$ profile. If we assume the $C_{3} / \Delta E$ profiles of other R-ratio cases are identical with the $C_{3} / \Delta E$ profile of the known $\mathrm{R}$-ratio case, which is true for this research, the $C_{3}$ and $\Delta V_{S E P S}$ can be extracted from the shifted $\Delta E$ profile and the extracted $\Delta V_{S E P S}$ can be put into the rocket equation solution to predict the delivered mass of other R-ratio cases. The reason why the $C_{\mathfrak{J}} / \Delta E$ profiles are almost identical in all missions can be explained as follow. Because all missions use

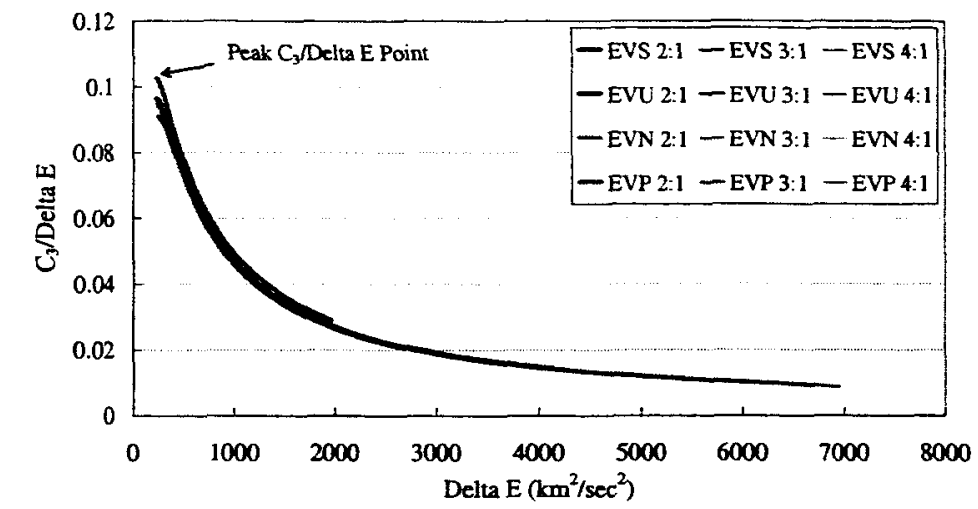

Figure 13. $C_{3} / \Delta E$ vs. $\Delta E$, short $T O F$, all R-ratios, all target missions.

the same launch vehicle, Delta IV M+(4,2) and the same SEPS (HTTP 3900 thruster), and the shapes of trajectories are similar except its R-ratio, the portion of $C_{3}$ (or $\Delta V_{S E P S}$ ) in $\Delta E$ profile, i.e. $C_{3} / \Delta E$ profile, are identical within an allowable error range for all target missions. Figure 14 illustrates the procedure of the hybrid estimation method for the short TOF range. Once we have a trajectory data for an R-ratio case from SEPTOP, the $\Delta E$ profile and the $C_{3} / \Delta E$ profile of the R-ratio case are available for the next step. By shifting the $\Delta E$ profile in TOF- $\Delta E$ plane, the $\Delta E$ profile of different R-ratio case can be approximated. The $\Delta V_{S E P S}$ is computed with the assumed $C_{3} / \Delta E$ profile and the definition of $\Delta E$. Then with the constant exhaust velocity from the constant thruster model and the $\Delta V_{S E P S}$, the delivered mass is computed for a different $\mathrm{R}$-ratio case without using SEPTOP. If the trajectories in different missions significantly differ from each other, then this assumption in the similarity of $C_{3} / \Delta E$ profile is not valid. In this research, however, all trajectories have similar characteristics except the different R-ratios in order to compare the effect of $R$-ratio to the delivered mass performance of a trajectory.

Another important application of the $C_{3} / \Delta E$ profiles is its peak point. The peak point is interpreted as the maximum relative usage of $C_{3}$ and there is a change in thrusting strategy in the left and right parts of the peak point. The peak point is equivalent to the beginning of stationary state of $\Delta E$ profile in Fig. 4

Because the $C_{3} / \Delta E$ profiles of other $\mathrm{R}$ ratio cases are assumed to be identical with the known $C_{3} / \Delta E$ profile, the peak $C_{3} / \Delta E$ points of other R-ratio cases are also identical with the known peak point. The peak point is used as the border between the short TOF range and the remaining TOF range in the hybrid method. Therefore, the method that uses the rocket equation solution is applied for the TOF $\leq$ peak TOF (the TOF value for the peak point) and the previous method that uses $k$ and $C$ is applied for the TOF $\geq$ peak TOF.

The $m_{0}$ in Eq. (15) can be calculated by a

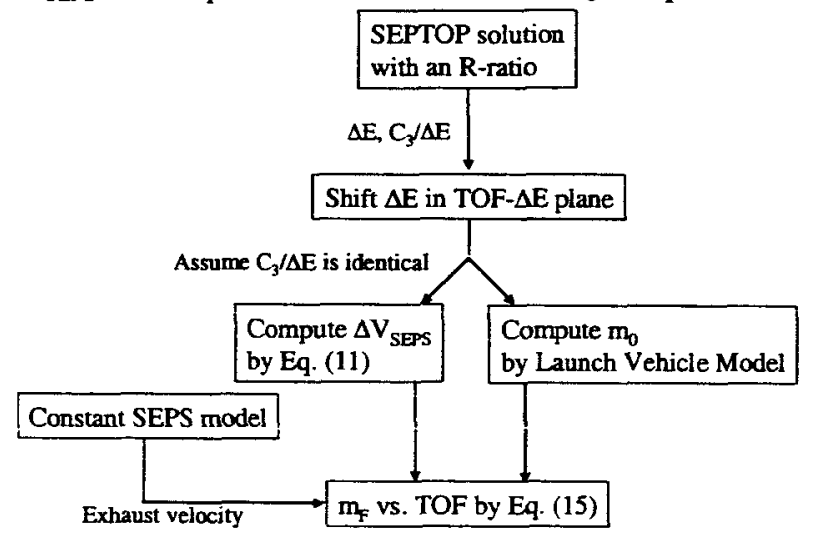

Figure 14. Procedure of hybrid method for short TOF range estimation.
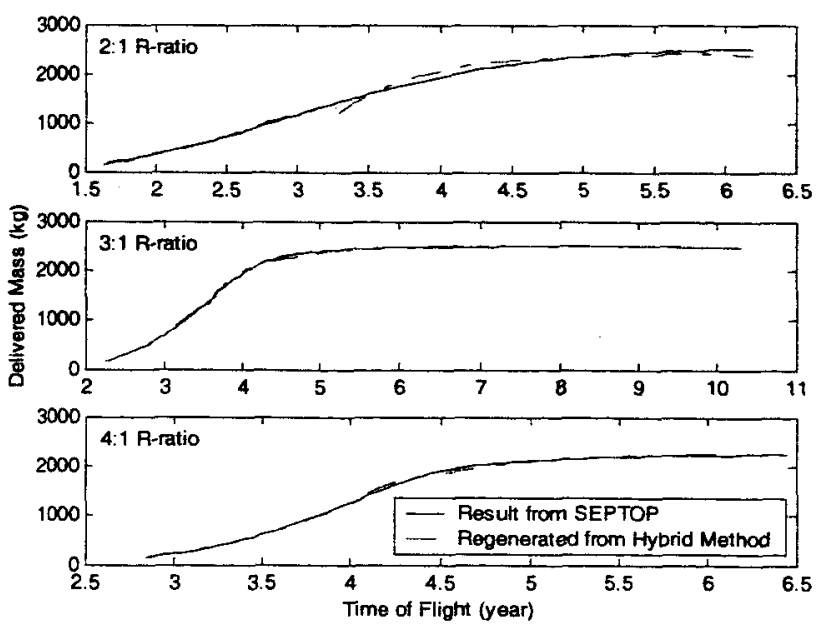

Figure 15. Hybrid Method Delivered mass comparison, the estimated versus SEPTOP, EVS mission. 
given launch vehicle model if $C_{3}$ is known. The $C_{3}$ and $\Delta V_{S E P S}$ can be extracted from the assumed $C_{3} / \Delta E$ profile and the shifted $\Delta E$ profile as previously explained. The exhaust velocity $c$ is not a constant for a SEPS but it is modeled as a constant with sufficient accuracy. Now, one can use the rocket equation solution to predict the delivered mass for the short TOF missions. The $\Delta E$ of 3:1 R-ratio case is chosen to be shifted and its $C_{3} / \Delta E$ is used for all other R-ratio cases. Figure 15 and Fig. 16 show the comparison between the estimated delivered mass curves and the delivered mass from SEPTOP for all R-ratio cases for EVS and EVP missions. The estimated delivered mass curves consist of two parts and their border is the peak TOF point of $C_{3} / \Delta E$ profile. It is clear that there is significant enhancement of the accuracy of the delivered mass estimation compared with Figs. 10 and 11 of the linear least square method. The maximum error in the short TOF range is less than $70 \mathrm{~kg}$ and the maximum error in the long TOF range is less than $100 \mathrm{~kg}$ for the hybrid method. For a comparison, the maximum error in the linear least square method was about $500 \mathrm{~kg}$. The reasons of this accuracy enhancement are first, the similarity of the $C_{3} / \Delta E$ profiles in short TOF range of the different R-ratio cases and second, the better linear least square fitting with smaller TOF range for the long TOF range. On the other hand, the error of the hybrid method comes from the assumptions about the $\Delta E$ profile, $C_{\mathfrak{y}} / \Delta E$ profile and the modeling error of the constant exhaust velocity of SEPS for the short TOF range. The incomplete symmetry between the delivered mass profile and the $\Delta E$ profile and the linear least square error are the sources of the errors in the longer TOF range.

\section{Conclusion}

Various R-ratio trajectories to outer planets are investigated in this paper. The trade-off between the launch energy and the onboard propulsive energy of SEPS for Venus gravity-assist missions is explained. An estimation method is presented for predicting mission performante for different R-ratio trajectories and can successfully regenerate the delivered mass profile only from one converged set of mission data and a given thruster model. Extensions of this method are currently being investigated.

\section{Acknowledgments}

The work described in this paper was funded in whole or in part by the In-Space Propulsion Technologies Program, which is managed by NASA's Office of Space Science in Washington, D.C., and implemented by the InSpace Propulsion Technology Projects Office at Marshall Space Flight Center in Huntsville, Ala. The program objective is to develop in-space propulsion technologies that can enable or benefit near and mid-term NASA space science missions by significantly reducing cost, mass and travel times.

\section{References}

${ }^{1}$ Strange, N. J., and Longuski, J. M., "Graphical Method for Gravity-Assist Trajectory Design," Journal of Spacecraft and Rockets, Vol. 39, No. 1, 2002, pp. 9-16.

${ }^{2}$ Woo, B., Coverstone, V., Hartmann, J., and Cupples, M., "Trajectory and Systems Analysis for Outer Planet Solar Electric Propulsion Missions," Journal of Spacecraft and Rockets, submitted for publication, 2004.

${ }^{3}$ Sauer, C. G., "Optimization of Multiple Target Electric Propulsion Trajectories," AIAA $11^{\text {th }}$ Aerospace Sciences Meeting, AIAA Paper 73-205, Washington, DC, Jan. 1973.

${ }^{4}$ Patterson, M., Foster, W. T., Rawlin, J. E., Roman, V., Robert, F., and Soulas, G., "Development Status of a 5/10-kW Class Ion Engine", $37^{\text {th }}$ Joint Propulsion Conference, AIAA 2001-3489, Salt Lake City, UT, July 8-11, 2001.

${ }^{5}$ Prussing, J. E. and Conway, B. A., Orbital Mechanics, Oxford University Press, New York, 1993, pp. 120-138.

${ }^{6}$ Sutton G. P. and Biblarz, O., Rocket Propulsion Elements, $6^{\text {th }}$ ed., John Wiley \& Sons, INC., New York, 2001, Chap. 12.

${ }^{7}$ Heath, M. T., Scientific Computing An introductory Survey Second Edition, McGraw Hill, Boston, 2002, pp. 120-131. 